\title{
Possibilidades da Investigação Conceitual/Filosófica na Análise do Comportamento
}

\author{
Alexandre Dittrich \\ Universidade Federal do Paraná
}

\begin{abstract}
RESUMO
Enquanto campo de investigação científica, a análise do comportamento é tradicionalmente caracterizada por uma divisão tripartite, que contempla análises conceituais/filosóficas, análises experimentais e análises aplicadas. $\mathrm{O}$ objetivo desse artigo é identificar e caracterizar as principais possibilidades de investigação no domínio das análises conceituais/filosóficas. A interpretação, definida como a extensão de princípios da análise do comportamento para a compreensão de relações comportamentais fora do âmbito experimental, é apresentada como um procedimento básico da análise conceitual/filosófica, em todas as suas modalidades. As possibilidades de investigação identificadas neste domínio são as seguintes: (1) sugerir à comunidade dos analistas do comportamento a adoção de certas posições epistemológicas e éticas consideradas coerentes e úteis; (2) dialogar com outras tradições de pensamento filosófico e científico (ou com autores específicos dentro dessas tradições); (3) ampliar o poder de compreensão e intervenção (experimental ou aplicada) da análise do comportamento sobre certos fenômenos comportamentais. Por fim, destaca-se que esta divisão não implica ou sugere uma separação estrita entre as diferentes possibilidades de investigação na análise conceitual/filosófica, e não esgota todas as possibilidades de investigação neste domínio.
\end{abstract}

Palavras-chave: análise do comportamento; behaviorismo radical; análise conceitual/filosófica; pesquisa em psicologia.

\begin{abstract}
Possibilities of Conceptual/Philosophical Investigation in Behavior Analysis

Behavior analysis, as a field of scientific investigation, is usually described as comprising a three-parts division: conceptual/philosophical analysis, experimental analysis and applied analysis. The objective of this article is to identify and characterize the main possibilities of investigation in the conceptual/philosophical analysis domain. Interpretation, defined as the extension of behavioranalytic principles to the comprehension of behavioral relations outside the experimental domain, is presented as a basic procedure in all kinds of conceptual/philosophical analysis. The possibilities of investigation identified in this domain are: (1) to suggest to the behavior-analytic community the adoption of certain epistemological and ethical positions, considered to be coherent and useful; (2) to communicate with other philosophical and scientific traditions (or with specific authors inside these traditions); (3) to increase the power to understand and intervene (in experimental and applied contexts) over certain behavioral phenomena. Finally, we point out that the proposed division does not imply or suggest a strict separation between the different possibilities of investigation in the domain of conceptual/philosophical analysis, neither exhausts all the possibilities of investigation in this domain.
\end{abstract}

Keywords: behavior analysis; radical behaviorism; conceptual/philosophical analysis; research in psychology.

O nome análise do comportamento tem sido genericamente utilizado para designar: (1) um empreendimento dedicado à produção de conhecimento em psicologia que compreende três domínios interligados de investigação: (a) análises conceituais/filosóficas, (b) análise experimental do comportamento, e (c) análise aplicada do comportamento; (2) um empreendimento dedicado à prestação de serviços psicológicos, visan- do a solução de problemas sociais relevantes através da utilização de técnicas comportamentais empiricamente validadas (Carvalho Neto, 2002; Hawkins \& Anderson, 2002; Moore \& Cooper, 2003; Tourinho, 1999b, 2003, 2006). Nesse artigo, dedicaremos especial interesse ao domínio (1-a). Cabem, porém, alguns esclarecimentos preliminares sobre os demais. 
Como nota Tourinho (2003, 2006), a análise do comportamento tem sido historicamente identificada com o domínio (1-b) - a análise experimental do comportamento. No campo mais amplo da análise do comportamento, ela cumpre o papel do que eventualmente recebe o nome de "pesquisa básica": trata-se de descrever relações funcionais generalizáveis entre (a) eventos ambientais manipulados pelo pesquisador e (b) respostas de um organismo, humano ou não. Resultam disso as "leis" ou "princípios" do comportamento, que nada mais são do que descrições econômicas das regularidades comportamentais observadas pelos cientistas em seus experimentos, com uma linguagem própria à sua comunidade científica.

O domínio (1-c) - a análise aplicada do comportamento - também se dedica a estudos empíricos, mas apresenta algumas características distintivas importantes em relação ao domínio (1-b). Enquanto a análise experimental do comportamento é, via de regra, realizada em laboratórios, a fim de permitir um controle tão preciso quanto possível das variáveis experimentais, a análise aplicada é realizada em ambientes sociais comuns. Na análise aplicada, além disso, os participantes são, via de regra, humanos ${ }^{1}$, enquanto na análise experimental as investigações envolvem várias espécies, incluindo humanos. Há, por fim, um interesse na análise aplicada em empregar princípios comportamentais já conhecidos (produzidos pela análise experimental) a fim de produzir resultados socialmente relevantes. Não se trata, portanto, de um empreendimento primordialmente voltado para a descoberta de novos princípios comportamentais, mas para a investigação sobre como tais princípios podem ser adequadamente aplicados para a solução de problemas sociais (Moore \& Cooper, 2003).

Analistas do comportamento têm notado, contudo, que a análise aplicada do comportamento apresenta diferenças importantes em relação ao que chamamos há pouco de empreendimento (2) - isto é, a utilização de técnicas analítico-comportamentais para a solução de problemas sociais (Hawkins \& Anderson, 2002; Moore \& Cooper, 2003). Essencialmente, essa diferença reside no fato de que no primeiro caso há um compromisso explícito com a pesquisa - isto é, com a coleta sistemática de dados e sua posterior apresentação à comunidade científica -, o que não se verifica no segundo caso, quando se trata apenas de prestar serviços fundamentados teoricamente na análise do comportamento. Isso não significa que no caso da prestação de serviços não haja coleta de dados, ou que ela não seja importante, mas certamente há diferenças relevantes nos objetivos e na forma de coleta de dados em cada caso. Hawkins e Anderson (2002), ao apontar algumas dessas diferenças, afirmam que, no caso da prestação de serviços, "o objetivo é uma mudança comportamental, não a explicação dessa mudança" (p. 118) - até porque o prestador de serviços lança mão de técnicas e princípios cuja efetividade já foi amplamente demonstrada em ocasiões anteriores (p. 117).

\section{Análises conceituais/filosóficas na análise do comportamento}

Resta-nos, enfim, abordar o domínio (1-a), referente às análises conceituais/filosóficas ${ }^{2}$. $\mathrm{Na}$ introdução do livro About Behaviorism, Skinner (1974) afirma que "o behaviorismo não é a ciência do comportamento humano; é a filosofia desta ciência" (p. 3). Do que trata essa filosofia? Tourinho (1999b) resume de forma adequada a proposta de Skinner: a filosofia behaviorista radical reflete sobre "o objeto, os objetivos e os métodos da ciência do comportamento" (p. 214). Utilizando a classificação filosófica comum, podemos afirmar que o behaviorismo radical trata de problemas epistemológicos/ontológicos (método e objeto) e éticos/políticos (objetivos).

A forma desse tratamento, porém, apresenta diferenças importantes em relação ao tratamento filosófico comum. Essas diferenças derivam, fundamentalmente, da adoção de uma forma particular de interpretação em relação aos fenômenos comportamentais. Skinner define interpretação como "o uso de termos e princípios científicos para falar de fatos sobre os quais se sabe muito pouco para tornar possíveis a previsão e o controle" (1984b, p. 578). As ferramentas interpretativas - isto é, os "termos e princípios científicos" aos quais se refere Skinner - são aqueles que, no âmbito da análise experimental do comportamento, se mostraram úteis para descrever relações comportamentais. A maioria das análises de Skinner sobre comportamentos humanos complexos (o comportamento verbal, as interações sociais, a auto-observação e o autocontrole) é interpretativa nesse sentido.

Todorov (1982) denomina tais análises interpretativas como "análises funcionais não-experimentais":

Muito do progresso obtido pela análise do comportamento deve-se a análises funcionais não experimentais. Seguramente, mais da metade dos escritos de Skinner refere-se a análises funcionais não-experimentais, isto é, à identificação (ou tentativa de) de variáveis dependentes e independentes, e de processos de interação em exemplos de comportamento humano. (p. 17) 
Para Vaughan e Michael (1982), “a função da interpretação é encorajar maiores extensões da análise comportamental, provendo uma explicação plausível a partir do esquema comportamental e, assim, gerar maiores desenvolvimentos experimentais, técnicos e teóricos" (p. 224).

A interpretação, assim definida, serve como um "pano de fundo" comum à maioria das investigações conceituais/filosóficas na análise do comportamento. A possibilidade de realizar tais interpretações permite, como dissemos há pouco, uma análise diferenciada dos problemas filosóficos tradicionais. Essa análise parte da constatação de que os problemas filosóficos dizem respeito, via de regra, ao comportamento humano. A epistemologia científica do behaviorismo radical, por exemplo, consiste, primordialmente, em uma análise interpretativa do comportamento dos cientistas e das comunidades científicas enquanto produtores de conhecimento. Essa análise inclui também o comportamento dos próprios analistas do comportamento. Zuriff (1980) identifica esse movimento afirmando que "uma ciência do comportamento inevitavelmente volta-se para dentro de si mesma" (p. 337).

O que resulta dessa análise interpretativa? Eis algumas afirmações possivelmente consensuais na análise do comportamento sobre a atividade científica: (1) Denominamos um ser humano "cientista" porque ele se comporta de determinadas formas sob certas circunstâncias, produzindo certas consequências. Um cientista é, simultaneamente, um organismo pertencente a uma espécie, uma pessoa com uma história de vida singular e um membro de certa cultura. Se ignorarmos qualquer um desses três conjuntos de fatores, não conseguiremos explicar porque um indivíduo qualquer se torna um cientista. (2) O comportamento do cientista é comportamento-em-contexto. Isso implica a interação do cientista com variáveis ambientais. Dessas variáveis, fazem parte não apenas os fenômenos estudados pelo cientista, mas também a cultura da qual o cientista faz parte, muito especialmente a comunidade verbal científica no interior da qual o cientista desenvolve suas atividades. (3) As práticas desta comunidade, por sua vez, devem ser compreendidas como resultantes de uma história e de um contexto cultural particular, assim como o comportamento individual dos cientistas. (4) A atividade científica é marcadamente verbal. O cientista segue, discute ou enuncia pressupostos filosóficos e regras metodológicas, analisa dados empíricos, discute e formaliza conceitos, leis e teorias.
Esta é uma análise propositadamente genérica do comportamento científico, mas ela ilustra uma forma comum de tratamento dos problemas filosóficos no behaviorismo radical. Para essa filosofia, a relação de conhecimento estabelecida entre o cientista e seu objeto de investigação é, por si mesma, um objeto de estudo legítimo no âmbito de uma ciência do comportamento (Abib, 1993; Skinner, 1945/1972, p. 380; 1963/1969, p. 228; 1974, p. 234-237; Zuriff, 1980). Como parte fundamental de sua epistemologia, interessa a um analista do comportamento saber o que faz um cientista, e por que o faz (inclusive quando qualifica certas afirmações como "verdadeiras"). Conhecer o mundo é comportar-se de certas formas em relação a ele - e, portanto, a atividade científica faz parte do campo de interesses do analista do comportamento. Ao perseguir esse interesse, repita-se, um analista do comportamento está interpretando - e ao fazê-lo, age como um filósofo behaviorista radical. $\mathrm{O}$ behaviorismo radical é uma filosofia psicológica: uma filosofia informada por dados produzidos pela análise do comportamento.

Assim como é possível realizar interpretações analítico-comportamentais de cunho epistemológico sobre o comportamento científico, é igualmente possível realizar tais interpretações sobre comportamentos verbais e não-verbais qualificados como "éticos" e "políticos" (Abib, 2002; Dittrich, 2004a, 2004b; Dittrich \& Abib, 2004; Garrett, 1979; Graham, 1977, 1983; Hocutt, 1977; Rottschaefer, 1980; Vargas, 1982; Waller, 1982). Essas interpretações constituem a base do que a filosofia behaviorista radical tem a dizer sobre ética e política. Alguns trabalhos discutem mais diretamente as consequências éticas e políticas das intervenções analítico-comportamentais (Dittrich, 2006; Fawcett, 1991; Holland, 1974, 1978; Task Force on Public Policy - Association for Behavior Analysis, 1988; Winett \& Winkler, 1972).

Embora a interpretação sirva, via de regra, como fundamento para as investigações conceituais/filosóficas na análise do comportamento, ela não garante, por si só, o cumprimento de algumas funções importantes contempladas por investigações desse tipo. A seguir, buscaremos especificar as principais:

(1) Como uma "filosofia crítica da ciência" (Skinner, 1963/1969, p. 267), o behaviorismo radical não se limita a propor uma análise interpretativa (e descritiva), por exemplo, do comportamento dos cientistas. $\mathrm{O}$ behaviorismo radical adota, a partir desta análise, uma epistemologia particular, com definições e conceitos próprios. Isso se expressa, por exemplo, no tratamento 
peculiar dispensado ao conceito de "verdade" (Barnes-Holmes, 2000; Hayes, 1993; Tourinho, 2003; Zuriff, 1980). As análises promovidas neste âmbito têm, para além de um papel descritivo, também um papel prescritivo, na medida em que buscam sugerir à comunidade dos analistas do comportamento a adoção de certas posições epistemológicas e éticas consideradas coerentes e úteis.

(2) O behaviorismo, como notou Skinner (1963/ 1969 , p. 221), é um "ismo" - isto é, um sistema particular de pensamento. Ele integra um universo intelectual que contém inúmeros outros "ismos", vários deles dedicados a responder perguntas semelhantes àquelas propostas pelo behaviorismo. Assim, é indispensável dialogar com esses outros "ismos", apontando quais deles caracterizam o behaviorismo radical, quais the são antitéticos, quais exerceram influência histórica em seu desenvolvimento, quais permitem intercâmbios produtivos, etc. ${ }^{5}$ Há um número considerável de trabalhos dedicados a essa tarefa (Abib, 1985, 2001; Lopes Jr., 1992; Moore, 1985; Morris, 1988, 1993; Slife, Yanchar, \& Williams, 1999; Smith, 1986; Tourinho, 1996, 1999a). Alguns trabalhos na mesma linha estabelecem diálogos com autores específicos dentro de certas tradições de pensamento, seja na filosofia ou nas ciências (Day, 1969; MacCorquodale, 1970; Marr, 2003; Morf, 1998; Smith, 1995; Tourinho \& Endemann, 2008).

(3) Análises conceituais podem ser feitas sem objetivos prioritariamente filosóficos (epistemológicos/ontológicos, éticos/políticos), mas visando ampliar o poder de compreensão e intervenção (experimental ou aplicada) da análise do comportamento sobre certos fenômenos comportamentais. Atualmente, os conceitos de metacontingência e macrocontingência constituem a melhor ilustração disso. Ambos contam com uma já longa história de refinamentos conceituais (Glenn, 1986, 1988, 1991, 2004; Malott \& Glenn, 2006), que tem sido acompanhada pelo crescimento de propostas de análises experimentais de análogos de metacontingências (Bullerjhann, 2009; Caldas, 2009; Leite, 2009; Oda, 2009; Pereira, 2008; Vichi, 2004). Considerando que tais propostas encontram fundamento nas próprias análises conceituais, esse exemplo dá conta do íntimo intercâmbio entre análises conceituais e empíricas na análise do comportamento. De modo geral, é possível constatar que exercícios de interpretação de fenômenos culturais através dos conceitos da análise do comportamento (incluindo ou não os conceitos de metacontingência ou macrocontingência) têm recebido atenção crescente (Guerin, 1994; Lamal, 1991; Lamal, 1997).
Relações comportamentais que contém eventos privados exigem esforços interpretativos especiais. A compreensão dos sonhos, por exemplo, tem demandado considerável esforço por parte de analistas do comportamento (Ferreira, 2006; Guilhardi, 1995; Melo e Silva, 2000). O conceito de atenção também pode ser citado nesse contexto (Strapasson \& Dittrich, 2008). Contudo, esses são apenas exemplos isolados do interesse maciço apresentado pelos analistas do comportamento na compreensão de relações comportamentais que contém eventos privados.

\section{CONCLUSÃO}

No início desse artigo, apresentamos a análise do comportamento, no que diz respeito à pesquisa, como "(1) um empreendimento dedicado à produção de conhecimento em psicologia que compreende três domínios interligados de investigação: (a) análises conceituais/filosóficas, (b) análise experimental do comportamento, e (c) análise aplicada do comportamento". Tourinho (1999b), contudo, faz notar que a caracterização de qualquer trabalho de pesquisa na análise do comportamento como pertencente exclusivamente a um desses três domínios é equivocada, pois

(...) um trabalho pode estar mais próximo de uma área de produção de conhecimento, mas cultivará sempre relações com as demais... A comunidade da análise do comportamento não promove e valida, por exemplo, estudos empíricos que não estão solidamente formulados com o aparato conceitual da área, ou modelos de intervenção que não se nutrem dos resultados derivados da investigação empírica. (p. 216-217)

Adicionalmente, é possível afirmar, sobre os estudos conceituais/filosóficos, que não serão promovidos e validados por esta comunidade caso não demonstrem coerência com os achados empíricos da análise experimental e aplicada, conforme sugere o próprio conceito de interpretação. Igualmente, não serão promovidos e validados se não demonstrarem alguma utilidade para a compreensão e intervenção da análise do comportamento sobre fenômenos comportamentais, ou, mais amplamente, para o aperfeiçoamento das práticas de ensino, pesquisa e intervenção empreendidas na análise do comportamento.

Uma diferenciação estrita entre estudos conceituais/filosóficos, experimentais e aplicados, aponta Tourinho (1999b), é não apenas difícil, mas indesejável. Possivelmente, o mesmo pode ser dito sobre a classificação há pouco proposta em relação às possibilida- 
des de trabalhos conceituas/filosóficos na análise do comportamento. Mesmo que se concentrem prioritariamente em uma das três possibilidades apontadas, com frequência trabalhos conceituais/filosóficos estabelecerão relações com as demais. Há pouco sentido, por exemplo, em estabelecer diálogos colaborativos com outros autores ou tradições de pensamento se tais diálogos não possibilitarem o enriquecimento ou refinamento dos conceitos e/ou propostas filosóficas da análise do comportamento.

A classificação proposta nesse artigo, portanto, tem sobretudo caráter analítico, e não implica ou sugere uma separação estrita entre as diferentes possibilidades de investigação na análise conceitual/filosófica. Tampouco esperamos que a classificação esgote todas as possibilidades de investigação neste domínio. Iniciativas no sentido de refinar e aperfeiçoar nossa proposta são não apenas possíveis, mas bem-vindas.

\section{REFERÊNCIAS}

Abib, J. A. D. (1985). Skinner, naturalismo e positivismo. Tese de doutorado não-publicada, Universidade de São Paulo.

Abib, J. A. D. (1993b). “A psicologia é ciência?" Ciência é articulação de discursos da filosofia, da história da ciência e da psicologia. Psicologia: Teoria e Pesquisa, 9, 465-486.

Abib, J. A. D. (2001). Behaviorismo radical como pragmatismo na epistemologia. Em H. J. Guilhardi, M. B. B. P. Madi, P. P. Queiroz \& M. C. Scoz (Orgs.), Sobre comportamento e cognição: Vol. 8. Expondo a variabilidade (pp. 158-161). Santo André, SP: ESETec.

Abib, J. A. D. (2002). Ética de Skinner e metaética. Em H. J. Guilhardi, M. B.B. P. Madi, P. P. Queiroz \& M. C. Scoz (Orgs.), Sobre comportamento e cognição: Vol. 10. Contribuições para a construção da teoria do comportamento (pp. 125137). Santo André, SP: ESETec.

Barnes-Holmes, D. (2000). Behavioral pragmatism: No place for reality and truth. The Behavior Analyst, 23, 191-202.

Bullerjhann, P. B. (2009). Análogos experimentais de fenômenos sociais: Os efeitos da consequência cultural. Dissertação de mestrado não-publicada, Pontifícia Universidade Católica de São Paulo.

Caldas, R. A. (2009). Análogos experimentais de seleção e extinção de metacontingências. Dissertação de mestrado nãopublicada, Pontifícia Universidade Católica de São Paulo.

Carvalho Neto, M. B. (2002). Análise do comportamento: Behaviorismo radical, análise experimental do comportamento e análise aplicada do comportamento. Interação em Psicologia, 6, 13-18.

Day, W. F. (1969). On certain similarities between the philosophical investigations of Ludwig Wittgenstein and the operationism of B. F. Skinner. Journal of the Experimental Analysis of Behavior, 12, 489-506.

De Rose, J. C. (1993). Classes de estímulos: Implicações para uma análise comportamental da cognição. Psicologia: Teoria e Pesquisa, 9, 283-303.
Dittrich, A. (2003). Introdução à filosofia moral skinneriana. Em C. E. Costa, J. C. Luzia \& H. H. N. Sant'Anna (Orgs.), Primeiros passos em análise do comportamento e cognição (pp. 1124). Santo André, SP: ESETec.

Dittrich, A. (2004a). A ética como elemento explicativo do comportamento no behaviorismo radical. Em M. Z. S. Brandão, F. C. S. Conte, F. S. Brandão, Y. K. Ingberman, V. L. M. Silva \& S. M. Oliani (Orgs.), Sobre comportamento e cognição: Vol. 13. Contingências e metacontingências: Contextos sócioverbais e o comportamento do terapeuta (pp. 21-26). Santo André, SP: ESETec.

Dittrich, A. (2004b). Behaviorismo radical, ética e política: Aspectos teóricos do compromisso social. Tese de doutorado, Universidade Federal de São Carlos. Retirado em 10 de dezembro de 2009, da Biblioteca Digital de Teses e Dissertações - BCo/UFSCar.

Dittrich, A. (2005). A atividade científica como objeto da ciência: Uma perspectiva contextualista behaviorista radical. Em Setor de Ciências Humanas, Letras e Artes - UFPR (Org.), Anais do III Encontro da Rede Paranaense de Pesquisa em História e Filosofia da Ciência (pp. 14-27). Curitiba: UFPR.

Dittrich, A. (2006). A sobrevivência das culturas é suficiente enquanto valor na ética behaviorista radical? Em H. J. Guilhardi \& N. C. Aguirre (Orgs.), Sobre comportamento e cognição: Vol. 17. Expondo a variabilidade (pp. 11-22). Santo André, SP: ESETec.

Dittrich, A., \& Abib, J. A. D. (2004). O sistema ético skinneriano e conseqüências para a prática dos analistas do comportamento. Psicologia: Reflexão e Crítica, 17, 427-433.

Fawcett, S. B. (1991). Some values guiding community research and action. Journal of Applied Behavior Analysis, 24, 621-636.

Ferreira, L. H. S. (2006). Pesquisa sobre interpretação de sonhos na análise do comportamento. Em H. J. Guilhardi \& N. C. Aguirre (Orgs.), Sobre comportamento e cognição: Vol. 17. Expondo a variabilidade (pp. 366-379). Santo André, SP: ESETec.

Garrett, R. (1979). Value conflict in a skinnerian analysis. Behaviorism, 7, 9-16.

Glenn, S. S. (1986). Metacontingencies in Walden Two. Behavior Analysis and Social Action, 5, 2-8.

Glenn, S. S. (1988). Contingencies and metacontingencies: Toward a synthesis of behavior analysis and cultural materialism. The Behavior Analyst, 11, 161-179.

Glenn, S. S. (1991). Contingencies and metacontingencies: Relations among behavioral, cultural, and biological evolution. Em P. A. Lamal (Org.), Behavioral analysis of societies and cultural practices (pp. 39-73). New York: Hemisphere.

Glenn, S. S. (2004). Individual behavior, culture, and social change. The Behavior Analyst, 27, 133-151.

Glenn, S. S., \& Malott, M. E. (2004). Complexity and selection: Implications for organizational change. Behavior and Social Issues, 13, 89-106.

Graham, G. (1977). On what is good: A study of B. F. Skinner's operant behaviorist view. Behaviorism, 5, 97-112.

Graham, G. (1983). More on the goodness of Skinner. Behaviorism, 11, 45-51.

Guerin, B. (1994). Analyzing social behavior: Behavior analysis and the social sciences. Reno: Context Press. 
Guilhardi, H. J. (1995). Um modelo comportamental de análise dos sonhos. Em B. Rangé (Org.), Psicoterapia comportamental e cognitiva de transtornos psiquiátricos (pp. 257-256). Campinas: Psy.

Hawkins, R. P., \& Anderson, C. M. (2002). On the distinction between science and practice: A reply to Thyer and Adkins. The Behavior Analyst, 25, 115-119.

Hayes, S. C. (1993). Analytic goals and the varieties of scientific contextualism. Em S. C. Hayes, L. J. Hayes, H. W. Reese \& T. R. Sabin (Orgs.), Varieties of scientific contextualism (pp. 1127). Reno: Context Press.

Hocutt, M. (1977). Skinner on the word "good": A naturalistic semantics for ethics. Ethics, 87, 319-338.

Holland, J. G. (1974). Are behavioral principles for revolutionaries? Em F. S. Keller \& E. Ribes-Inesta (Orgs.), Behavior modification: Applications to education (pp. 195-208). New York: Academic Press.

Holland, J. G. (1978). Behaviorism: Part of the problem or part of the solution? Journal of Applied Behavior Analysis, 11, 163174.

Lamal, P. A. (Org.) (1991). Behavioral analysis of societies and cultural practices. New York: Hemisphere.

Lamal, P. A. (Org.) (1997). Cultural contingencies: Behavior analytic perspectives on cultural practices Westport, CT: Praeger.

Leite, F. L. (2009). Efeitos de instruções e história experimental sobre a transmissão de práticas de escolha em microculturas de laboratório. Dissertação de mestrado não-publicada, Universidade Federal do Pará, Belém.

Lopes Jr., J. (1992). Sobre os critérios de interpretação da história do behaviorismo radical (1930-1945): Operacionismo e privacidade. Dissertação de mestrado não-publicada, Universidade de São Paulo.

MacCorquodale, K. (1970). On Chomsky's review of Skinner's verbal behavior. Journal of the Experimental Analysis of Behavior, 13(1), 83-99.

Malott, M. E., \& Glenn, S. S. (2006). Targets of intervention in cultural and behavioral change. Behavior and Social Issues, 15, 31-56.

Marr, M. J. (2003). The what, the how, and the why: The explanation of Ernst Mach. Behavior and Philosophy, 31, 181-192.

Melo e Silva, F. (2000). Uma análise behaviorista radical dos sonhos. Psicologia: Reflexão e Crítica, 13, 435-449.

Morf, M. E. (1998). Sartre, Skinner and the compatibilist freedom to be authentically. Behavior and Philosophy, 26, 29-43.

Moore, J. (1985). Some historical and conceptual relations among logical positivism, operationism, and behaviorism. The Behavior Analyst, 8, 53-63.

Moore, J., \& Cooper, J. O. (2003). Some proposed relations among the domains of behavior analysis. The Behavior Analyst, 26, 69-84.

Morris, E. K. (1988). Contextualism: The world view of behavior analysis. Journal of Experimental Child Psychology, 46, 289323.

Morris, E. K. (1993). Behavior analysis and mechanism: One is not the other. The Behavior Analyst, 16, 25-43.

Oda, L. V. (2009). Investigação das interações verbais em um análogo experimental de metacontingência. Dissertação de mestrado não-publicada, Pontifícia Universidade Católica de São Paulo.

Pereira, J. M. C. (2008). Investigação experimental de metacontingências: Separação do produto agregado e da conseqüência individual. Dissertação de mestrado não-publicada, Pontifícia Universidade Católica de São Paulo.

Rottschaefer, W. A. (1980). Skinner's science of value. Behaviorism, 8, 99-112.

Skinner, B. F. (1965). Science and human behavior. New York: Macmillan. (Original publicado em 1953)

Skinner, B. F. (1969). Behaviorism at fifty. Em B. F. Skinner (Org.), Contingencies of reinforcement: A theoretical analysis (pp. 221-268). New York: Appleton-Century-Crofts. (Original publicado em 1963)

Skinner, B. F. (1969). Behaviorism at fifty. Em B. F. Skinner (Org.), Contingencies of reinforcement: A theoretical analysis (pp. 221-268). New York: Appleton-Century-Crofts. (Original publicado em 1963)

Skinner, B. F. (1971). Beyond freedom and dignity. New York: Alfred A. Knopf.

Skinner, B.F. (1972). The operational analysis of psychological terms. Em B.F. Skinner (Org.), Cumulative record: A selection of papers (pp. 370-384). New York: Appleton-Century-Crofts. (Original publicado em 1945)

Skinner, B. F. (1974). About behaviorism. New York: Alfred A. Knopf.

Skinner, B.F. (1984a). A matter of consequences: Part three of an autobiography. New York University Press. (Original publicado em 1983)

Skinner, B. F. (1984b). Coming to terms with private events. The Behavioral and Brain Sciences, 7, 572-579.

Slife, Yanchar, \& Williams (1999). Conceptions of determinism in radical behaviorism: A taxonomy. Behavior and Philosophy, 27, 75-96.

Smith, L. D. (1986). Behaviorism and logical positivism: A reassessment of the alliance. Stanford: Stanford University Press.

Smith, L. D. (1995). Inquiry nearer the source: Bacon, Mach, and The Behavior of Organisms. Em J. T. Todd \& E. K. Morris (Orgs.), Modern perspectives on B. F. Skinner and contemporary behaviorism (pp. 39-50). Westport: Greenwood Press.

Strapasson, B. A., \& Dittrich, A. (2008). O conceito de "prestar atenção" para Skinner. Psicologia: Teoria e Pesquisa, 24, 519526.

Task Force on Public Policy - Association for Behavior Analysis (1988). Recommendations of the Task Force on Public Policy. The Behavior Analyst, 11, 27-32.

Todorov, J. C. (1982). Behaviorismo e análise experimental do comportamento. Cadernos de Análise do Comportamento, 3, $10-23$.

Tourinho, E. Z. (1996). Behaviorismo radical, representacionismo e pragmatismo. Temas em Psicologia, 2, 41-56.

Tourinho, E. Z. (1999a). Consequências do externalismo behaviorista radical. Psicologia: Teoria e Pesquisa, 15, 107-115.

Tourinho, E. Z. (1999b). Estudos conceituais na análise do comportamento. Temas em Psicologia, 7, 213-222.

Tourinho, E. Z. (2003). A produção de conhecimento em psicologia: A análise do comportamento. Psicologia: Ciência e Profissão, 23, 30-41. 
Tourinho, E. Z. (2006). Relações comportamentais como objeto da psicologia: Algumas implicações. Interação em Psicologia, 10, $1-18$.

Tourinho, E. Z., \& Endemann, P. (2008). Linguagem e conhecimento em B. F. Skinner e J. L. Austin. Acta Comportamentalia, 16, 17-137.

Tourinho, E. Z., \& Neno, S. (2003). Effectiveness as truth criterion in behavior analysis. Behavior and Philosophy, 31, 63-81.

Vargas, E. A. (1982). Hume's "ought" and "is" statement: A radical behaviorist perspective. Behaviorism, 10, 01-23.

Vaughan, M. E., \& Michael, J. L. (1982). Automatic reinforcement: An important but ignored concept. Behaviorism, 10, $217-$ 227.
Vichi, C. (2004). Igualdade ou desigualdade em pequeno grupo: Um análogo experimental de manipulação de uma prática cultural. Dissertação de mestrado não-publicada, Pontifícia Universidade Católica de São Paulo.

Waller, B. (1982). Skinner's two stage value theory. Behaviorism, $10,25-44$.

Winett, R. A., \& Winkler, R. C. (1972). Current behavior modification in the classroom: Be still, be quiet, be docile. Journal of Applied Behavior Analysis, 5, 499-504.

Zuriff, G. E. (1980). Radical behaviorist epistemology. Psychological Bulletin, 87, 337-350.

Notas:

1 Moore e Cooper (2003), contudo, apontam exceções - como, por exemplo, o treinamento de animais para auxiliar pessoas cegas (p. 76).

2 Propositadamente, não trataremos de trabalhos de cunho histórico, pois estes exigem métodos diferenciados em relação às análises conceituais/filosóficas. Não há dúvidas, porém, sobre a utilidade de tais trabalhos para as análises conceituais/filosóficas. Tourinho (1999), por exemplo, ao discorrer sobre estudos conceituais/filosóficos na análise do comportamento, afirma que há neles, “invariavelmente, um conteúdo histórico, na medida em que são referenciados pelo processo permanente de (re)elaboração do sistema explicativo" (p. 213).

3 Em Beyond Freedom and Dignity, encontramos uma definição semelhante: "Muitas vezes referimo-nos a coisas que não podemos observar ou medir com a precisão demandada por uma análise científica, e, ao fazê-lo, há muito a se ganhar ao usar termos e princípios que foram forjados em condições mais precisas" (Skinner, 1971, pp. 22-23). Dito de outra forma: onde a experimentação não é possível, utilize-se a interpretação - mas uma interpretação baseada em resultados experimentais. A interpretação, assim definida, não é uma exclusividade da análise do comportamento, mas uma prática comum e necessária também nas demais ciências (Skinner, 1974, p. 19).

4 Em outros trabalhos (Dittrich, 2004b, 2005), ampliamos a análise sobre o comportamento científico, e abordamos alguns problemas gerados pela proposta de uma filosofia psicológica. Por ora, cabe notar, pelo menos, que (1) o behaviorismo radical não tem a pretensão de enunciar verdades fundamentais, imutáveis, indiscutíveis sobre os assuntos que aborda; (2) mesmo entre os próprios behavioristas radicais os resultados das interpretações comportamentais são variáveis e discutíveis (o que é bem ilustrado pelos estudos sobre ética e política citados no parágrafo seguinte).

5 Deve-se notar, porém, que a própria necessidade ou importância das classificações filosóficas pode ser questionada - e mesmo o texto skinneriano fornece subsídios para tanto. Ao comentar sobre "os perigos inerentes em qualquer sistema de tipologia", Skinner afirma: "Há sempre uma tendência a argumentar que, porque indivíduos são similares em um aspecto, eles também são similares em outros" (1953/1965, p. 424). Em sua autobiografia, Skinner relata um encontro com Henry Kissinger, no qual este teria atribuído as dificuldades de relacionamento entre americanos e russos ao fato de seus governos serem, respectivamente, democrático e revolucionário. Diante disso, Skinner relata: "Eu disse que achava as tipologias perigosas; na psicologia, elas foram devastadoras" (1983/1984a, p. 151). Contudo, mesmo que concordemos com as considerações de Skinner sobre o assunto, pode-se afirmar que as tipologias ou classificações - isto é, os "ismos" - são, no mínimo, um mal necessário. Os "ismos" também são conceitos, e talvez o próprio pensar fosse difícil ou impossível sem eles (vide, por exemplo, a abordagem sobre a formação de conceitos em de Rose, 1993). Para além de sua inevitabilidade, contudo, é possível apontar em tais classificações algumas características úteis. Como aponta Abib (1985), as classificações filosóficas são importantes "porque chamam nossa atenção para alguns aspectos que às vezes passam despercebidos", ressaltando-se, porém, que "o ponto importante é que não devemos nunca perder de vista a totalidade da obra do autor"' (p. 203).

\section{Sobre o autor:}

Alexandre Dittrich - Psicólogo, Doutor em Filosofia pela Universidade Federal de São Carlos. Professor do Departamento de Psicologia da Universidade Federal do Paraná.

Endereço eletrônico: aledittrich@ufpr.br. 